\title{
Acknowledgement to Reviewers of Humanities in 2020
}

\section{Humanities Editorial Office}

Citation: Humanities Editorial Office. 2021. Acknowledgment to

Reviewers of Humanities in 2021.

Humanities 10: 24.

https://doi.org/10.3390/h10010024

Published: 1 February 202

Publisher's Note: MDPI stays neutral with regard to jurisdictional claims in published maps and institutional affiliations.

Copyright: (C) 2021 by the author. Licensee MDPI, Basel, Switzerland. This article is an open access article distributed under the terms and conditions of the Creative Commons Attribution (CC BY) license (http://creativecommons.org/licenses /by/4.0/).

MDPI AG, St. Alban-Anlage 66, 4052 Basel, Switzerland

Peer review is an essential part in the publication process, ensuring that Humanities maintains high quality standards for its published papers. In 2020, a total of 134 papers were published in the journal. Thanks to the cooperation of our reviewers, the median time to first decision was 20.5 days and the median time to publication was 62.5 days. The editors would like to express their sincere gratitude to the following reviewers for their time and dedication in 2020:

Abdullahi, Ibrahim

Abhik, Chakraborty

Ad, Putter

Adam, Hart

Adele, Newson-Horst

Adrian, Favell

Agnieszka, Poczta-Wajda

Aike Peter, Rots

Aimee, Pozorski

Alec, Cattell

Aleta-Amirée, Von Holzen

Alexandra, Watkins

Alfonso, Ballesteros

Alisa Ballard, Lin

Alison, Booth

Almas, Khan

Amanda, Lagji

Amedeo, Policante

Ana, Amante

Ananda, Abeysekara

Andrea, Hunt

Andrea, Severson

Andreas, Dix

Andrew, $\mathrm{Ng}$

Anna, Branach-Kallas

Anna, Kotova

Anna-Katharina, Wöbse

Anne, McConnell

Annouchka, Bayley

Anthony Kwame, Harrison

Anton, Kirchhofer

Antonella, Castelvedere

Antonio, Alcala-Gonzalez

Asim, Ali

Axel, Goodbody
Axel, Stähler

Babacar, M'Baye

Bart, Welling

Benjamin, Meade

Bernhard, Malkmus

Bozena, Karwowska

Brad, Prager

Brent Ryan, Bellamy

Brenton, Dickieson

Bruce, McNair

Bruce, Plourde

Callie, Watkins Liu

Carmelina, Concilio

Caroline, Rosenthal

Caroline, Tully

Carolyn, Oulton

Cassandra, Chaney

Caterina, Colomba

Catherine L., Benamou

Catrin, Webster

Chad, Weidner

Chielozona, Eze

Chris, Hill

Christine A., James

Christine Knoblauch, O'Neal

Christine, Berberich

Christine, Lorre-Johnston

Christine, Okoth

Christopher, Breu

Christopher, Holliday

Christopher, Siwicki

Clayton, Crockett

Clive, Cazeaux

Colette, Balmain

Colin, Herd 
Constant, Mews

Cristóbal Macías, Villalobos

Cynthia, Hogan

Dagmara, Drewniak

Dan, Ben-Amos

Dana, Mihailescu

Daniel, Finch-Race

Danielle, Spratt

Danine, Farquharson

Dara, Downey

David V., Urban

David, Arnold

David, Calonne

David, Haney

David, Strong

Davis, Schneiderman

Deirdre, Osborne

Denise, Grollmus

Diana, Barnes

Dolores, Herrero

Donald R., Wehrs

Doris, Frye

Dorothee, Kimmich

Doug, Davis

Ed, Cameron

Edward, Howell

Elana, Gomel

Eleanor, Novek

Elena, Ioannidou

Eleonora, Rao

Elizabeth Lane Williams, Ørberg

Elizabeth, Greene

Elizabeth, Gregory

Elizabeth, Hoiem

Elizabeth, Kella

Emilia, Nielsen

Emily, Kieson

Emily-Rose, Baker

Enrique, Pastor Seller

Eric, Leuschner

Eric, Schaefer

Erik, Mortenson

Esther, Clinton

Eugene, Arva

Evan, Carver

Ewa, Stańczyk

Eyal, Lewin

Fiona, Nicoll

Francesc, Miralles

Francesca, Bargiela-Chiappini

Franz, Winter
Fred, Sutherland

Frederike, Middelhoff

Fredrik, Thue

Gabriele, Dürbeck

Gareth, Knapman

Gerd, Bayer

Giovanni, Messina

Giovanni, Peira

Godela, Weiss-Sussex

Grace C., Tiffany

Gregory M., Clines

Grigore, Herman

Grzegorz, Rosslinski-Liebe

Hadeel, Abdelhameed

Hala Ibrahim, Ghoneim

Hannah, Holtschneider

Hannu, Heikkinen

Hans-Peter, Ecker

Harvey C., Mansfield

Heather, Sullivan

Helen Conrad, O'Briain

Helen, Finch

Helen, Parish

Helena, Woodard

Helga G., Braunbeck

Henrik, Johnsson

Hiromasa, Tanaka

Ian, Davidson

Iftikhar, Malik

Ignasi, Ribó

Inês, Amorim

Irina Igorevna, Strout

Iris, Van Der Tuin

Isaac, Land

Isak, Hammar

Izabella, Kimak

Jacob, McGuinn

Jacquelene G., Brinton

Jad, Smith

James, Hatley

Janet M., Wilson

Jason Baird, Jackson

Jayne, Finlay

Jeanne, Dubino

Jeff, Tischauser

Jeffery D., Long

Jennie, Klein

Jennifer, Cognard-Black

Jennifer, Hansen-Glucklich

Jessica, Aliaga-Lavrijsen

Jesús David, Jerez-Gómez 
Jill Terry, Rudy

Jimmy, Packham

Joanna, Kruczkowska

Joanne, Leal

Jochen, Petzold

Joela, Jacobs

John, Buckridge

John, Michael

Jolene, Zigarovich

Jonathan Blake, Fine

Joni, Adamson

Josef, Zelenka

Joseph, Brown

Joseph, Thorne

Joseph, Zompetti

Joshua, Miller

Joy Kreeft, Peyton

Judy Tydor, Baumel-Schwartz

June, McDaniel

Kamil, Kopania

Kate, Aughterson

Kate, Eichhorn

Katharina, Bonzel

Katia, Garloff

Katrina, Hinson

Keith, Moser

Kelebogile T., Resane

Keren, Omry

Kerstin, Germer

Kirsten, Lodge

Kurt, Hemmer

Kyle, Bishop

Laura, Bradley

Laura, Winkiel

Lavonna L., Lovern

Lee, Wallace

Leonard, Neidorf

Ligita, Baležentienè

Linda Kinsey Adams, Spetter

Linda, Belau

Linus, Salö

Lisa, Han

Liz, McDowell

Lizzie, Stewart

Lorenzo, Veracini

Lucy, Arnold

Lukas, Engelmann

Luke, Bennett

Lynette, Goddard

Macklin, Rebecca

Magdalena, Czalczynska-Podolska
Magdalena, Wojnowska-Heciak

Mandy, Bloomfield

Manuel Jesús, Cardoso Pulido

Marcia, Morgan

Margarette, Lincoln

Maria Candida, Ghidini

María Carmen Africa, Vidal Claramonte

Maria, Medina-Vicent

Mark, Llewellyn

Mark, McInroy

Mark, Sandy

Mark, Watson

Marko, Pajevic

Mary Catherine, Kinniburgh

Mary Ellen, Mastrorilli

Mary, Christian

Mary, Keller

Matt, Marostica

Matthew, Chrulew

Matthew, Crofts

Matthew, Nelson

Matthias, Pirholt

Maureen Daly, Goggin

Maureen, Reddy

Melanie, Connor

Merel A., Visse

Michael, Bryson

Michael, Meyer

Michael, Niblett

Michaela, Raggam-Blesch

Michelle, Slater

Mila, Ganeva

Minlib, Dallh

Minna, Vuohelainen

Min-seong, Kim

Nana, Amoah-Ramey

Nancy, Holmes

Nicholas, Birns

Niklas, Salmose

Nina, Engelhardt

Noah, Benninga

Nora, Pleßke

Norman, Smith

Norman, Vance

Oliver C. G., Harris

Otto, Heim

Paola, Botham

Paul, Holloway

Paul, March-Russell

Paulo, Matos

Pekka, Hakamies 
Peter E., Cumming

Peter, Verstraten

Philip Edward, Phillips

Philip, McGowan

Phillip, Zapkin

Pilar, Royo-Grasa

Pippa, Marland

Rebeca, Pardo

Rebecca Futo, Kennedy

Rebecca, Duncan

Reighan, Gillam

Rhiannon M., Cates

Rita, Keresztesi

Rita, Salvi

Rob, Pensalfini

Robert, Kellerman

Robert, Mugerauer

Robert, Prentner

Roberta, Dreon

Roberto, Baelo

Robin, Armstrong

Ronald Michael, Green

Rossella, Ciocca

Rowan, Mackenzie

Ruth Bienstock, Anolik

Ruth, Maxey

Saba, Pirzadeh

Sabine, Egger

Sally L., Kitch

Sarah Juliet, Lauro

Sarah, Brouillette

Sarah, Coakley

Scott, Slovic

Sean, Moreland

Seb, Coxon

Shamika Ann, Mitchell

Shannon R., Wooden

Sharon, Jones

Sharon, Oster

Sheila, Cavanagh

Silvia, Castro Borrego
Silvia, Nobre

Silvia, Pellicer-Ortín

Simon, Kemp

Simona, Mitroiu

Simone, Guidi

Sofia, Cavalcanti

Sonja, Hedgepeth

Stefan, Silber

Stephanie, Callan

Stephanie, Jones

Steven, Rawle

Sue, Vice

Susan, Morrison

Susan, Watkins

Susana, Onega Jaén

Suzanne, Keen

Teresa, Seruya

Thomas, Bristow

Thomas, Craemer

Thomas, Tartaron

Timothy C., Baker

Timothy S., Miller

Tom, Havens

Tom, Smith

Tomasz, Łysak

Treasa De, Loughry

Unnur, Ottarsdottir

Ursula, Kluwick

Vernon, Shetley

Victoria, Aarons

Vinicius De, Carvalho

W. Dana, Phillips

Wendy, Hinshaw

Wieland, Schwanebeck

Wieslaw, Ziaja

Will, Lombardi

Will, May

Xavier, Marquez

Yeo Kah Sin, Dennis

Yosef, Schwartz

Zsófia, Gombár 